\title{
REAL SECTORIAL OPERATORS
}

A. Yagi, Department of Applied Physics, Osaka University, Suita, Osaka, Japan, yagi@ap.eng.osaka-u.ac.jp

Sectorial operators that act in complex Banach spaces and map real subspaces into themselves should be called real sectorial operators. These operators have already been used implicitly in the study of various diffusion equations. Meanwhile, in the EojasiewiczSimon theory which provides longtime convergence of solutions to stationary solutions, the real valued Lyapunov functions play an important role. In order to make general methods for studying longtime convergence problems on the basis of the Eojasiewicz Simon theory, it may therefore be meaningful to give an explicit definition for these real sectorial operators and to show their basic properties that are inherited from those of complex sectorial operators.

Keywords: sectorial operators; fractional powers of operators; differential operators.

Dedicated to Professor Angelo Favini on the occasion of his 70th birthday.

\section{Introduction}

When we want to study diffusion equations, there is an advantage of handling them in complex valued function spaces than in real valued function spaces, because the second order elliptic operators often generate an analytic (holomorphic) semigroup in a suitable complex Banach space. Using the techniques of functional analysis including such analytic semigroups, construction of local or global solutions can easily be carried out, see Krein [1], Tanabe [2], Favini, Yagi [3] and Yagi [4]. In the meantime, unknown functions of diffusion equations often denote densities or concentrations of some physical objects or chemical substances and they are real valued. Thereby, only real parts of unknown functions are meaningful in applications.

Fortunately, when diffusion equations are well posed, the solutions are always real if their initial functions are real. This fact means that one can construct desired real solutions in the framework of complex function spaces. Even more, as seen by [5,6] for example, one can use the Łojasiewicz - Simon theory in order to prove longtime convergence of real solutions to stationary solutions. Furthermore, the arguments in $[5,6]$ suggest that one could make general methods for studying the longtime convergence problems in a unified way for various diffusion equations employing the Łojasiewicz - Simon theory. To the ends, however, we have to begin by constructing firm frameworks.

In the Łojasiewicz - Simon theory, the gradient inequality (see Chill [7] and Haraux, Jendoubi [8]) for the Fréchet derivatives of real valued Lyapunov functions plays a crucial role. So, everything must be set in the sense of "real", namely, real Banach spaces, real sectorial operators, real analytic semigroups, interpolations of real Banach spaces by the complex methods, and so on. As a matter of fact, these things have already been used implicitly in the various complex settings. The objectives of this Note are then to introduce an explicit definition of real sectorial operators acting in the real subspaces and 
to show their basic properties which are reasonably analogous to those of complex sectorial operators.

When a densely defined, closed linear operator acting in a complex Banach space has its spectrum contained in a sectorial complex domain and satisfies an optimal decay estimate of resolvent, the operator is called a sectorial operator (Brezis [9] and Yosida [10]). This notion has naturally been defined only for complex linear operators. Meanwhile, when an underlying complex Banach space $\widetilde{X}$ admits a conjugation $f \mapsto \bar{f}$ and is decomposed into $\widetilde{X}=X+i X$, where $X$ is a real Banach subspace of $\widetilde{X}$, and when a sectorial operator $A$ of $\tilde{X}$ maps $\mathcal{D}(A) \cap X$ into $X$, we call its part $A_{\mid X}$ restricted in $X$ a real sectorial operator induced from $A$. It is verified that $A_{\mid X}$ inherits basic properties from $A$.

\section{Complex Banach Spaces with Conjugation}

We begin with defining conjugation acting on a complex Banach space. Let $\widetilde{X}$ be a complex Banach space with norm $\|\cdot\|$. Assume that $\widetilde{X}$ is equipped with a correspondence $f \mapsto \bar{f}$ satisfying:

$$
\begin{gathered}
\overline{f+g}=\bar{f}+\bar{g} \quad \text { for } f, g \in \tilde{X}, \\
\overline{\alpha f}=\bar{\alpha} \bar{f} \quad \text { for } \quad \alpha \in \mathbb{C}, f \in \widetilde{X}, \\
\overline{\bar{f}}=f \quad \text { for } f \in \widetilde{X}, \\
\|\bar{f}\|=\|f\| \quad \text { for } f \in \widetilde{X} .
\end{gathered}
$$

It is immediate to verify that the correspondence is continuous on $\widetilde{X}$ and one-to-one and onto. In particular, $\overline{0}=0$. The vector $\bar{f}$ is called the conjugate vector of $f$. Such a correspondence is called a conjugation on the space $\widetilde{X}$.

For $f \in \widetilde{X}$, we put

$$
\operatorname{Re} f=\frac{f+\bar{f}}{2} \quad \text { and } \quad \operatorname{Im} f=\frac{f-\bar{f}}{2 i} .
$$

Then, it is clear that $f=\operatorname{Re} f+i \operatorname{Im} f$. The vector $\operatorname{Re} f($ resp. $\operatorname{Im} f)$ is called the real part (resp. imaginary part) of $f \in \widetilde{X}$. The vectors satisfying $\operatorname{Im} f=0$ or equivalently $\bar{f}=f$ are called a real vector. By (1), (2) and (3), both $\operatorname{Re} f$ and $\operatorname{Im} f$ are a real vector. As noticed, 0 is also a real vector. On the other hand, the vectors satisfying $\operatorname{Re} f=0$ or equivalently $\bar{f}=-f$ are called a purely imaginary vector. Obviously, $i \operatorname{Im} f$ is a purely imaginary vector. It holds that

$$
\overline{\operatorname{Re} f+i \operatorname{Im} f}=\operatorname{Re} f-i \operatorname{Im} f \quad \text { for } f \in \widetilde{X} .
$$

We want to consider the space

$$
X=\{f \in \widetilde{X} ; \operatorname{Im} f=0 \text {, i.e., } \bar{f}=f\} .
$$

By (1) and (2), $X$ is a real vector space equipped with the norm $\|\cdot\|$. We see the following fact.

Theorem 1. $X$ is a closed subset of $\widetilde{X}$ and is a real Banach space.

Proof. By definition, $X=\operatorname{Im}^{-1} 0$. Meanwhile, $f \mapsto \operatorname{Im} f$ is continuous; therefore, $X$ is a closed subset. 
Since $X$ is a real normed space, it suffices to verify its completeness. But, as $X$ is a closed subset of a complete space $\widetilde{X}, X$ is naturally complete.

We call $X$ the real Banach subspace induced from $\tilde{X}$.

Thereby, we have a decomposition of any $f \in \widetilde{X}$ into the form

$$
f=\operatorname{Re} f+i \operatorname{Im} f \quad \text { with } \quad \operatorname{Re} f, \operatorname{Im} f \in X .
$$

Indeed, such a decomposition is unique.

Theorem 2. For any $f \in \tilde{X}, f$ can be written as $f=f_{1}+i f_{2}, f_{k} \in X(k=1,2)$ in a unique way. The correspondence $f \mapsto f_{k}$ is continuous from $\widetilde{X}$ onto $X$ for $k=1,2$. In this sense, $\widetilde{X}=X+i X$.

Proof. Since (7) gives such a decomposition, it is sufficient to prove the uniqueness. Let $f=f_{1}+i f_{2}=g_{1}+i g_{2}$ with $f_{k}, g_{k} \in X$ for $k=1,2$. Then, $\left(f_{1}-g_{1}\right)+i\left(f_{2}-g_{2}\right)=0$; at the same time, considering this conjugate, we have $\left(f_{1}-g_{1}\right)-i\left(f_{2}-g_{2}\right)=0$. Therefore, $f_{1}=g_{1}$ and $f_{2}=g_{2}$. Hence, (7) is the only possible decomposition.

By (4), we see that

$$
\|f\| \leq\|\operatorname{Re} f\|+\|\operatorname{Im} f\| \leq 2 \max \{\|\operatorname{Re} f\|,\|\operatorname{Im} f\|\} \leq 2\|f\|, \quad f \in \widetilde{X} .
$$

This readily yields that $f \mapsto \operatorname{Re} f$ and $f \mapsto \operatorname{Im} f$ are continuous from $\tilde{X}$ onto $X$.

Corollary 1. When $\widetilde{X}$ is a complex Hilbert space with inner product $(\cdot, \cdot)$, its real Banach subspace $X$ is a real Hilbert space with the same inner product.

Proof. Since

$$
4(f, g)=\|f+g\|^{2}-\|f-g\|^{2}+i\|f+i g\|^{2}-i\|f-i g\|^{2},
$$

it follows that

$$
\overline{4(f, g)}=\|f+g\|^{2}-\|f-g\|^{2}-i\|f+i g\|^{2}+i\|f-i g\|^{2} .
$$

In view of (4), we observe that

$$
\overline{(f, g)}=(\bar{f}, \bar{g}) \quad \text { for } f, g \in \tilde{X} .
$$

If $f, g \in X$, then $\overline{(f, g)}=(f, g)$. Thus, $(\cdot, \cdot)$ defines a real inner product on $X$ which provides a Hilbert structure.

\section{Real Sectorial Operators}

We now state a definition of real sectorial operators. Let $\widetilde{X}$ be a complex Banach space with norm $\|\cdot\|$. Assume that $\widetilde{X}$ is equipped with a conjugation $f \mapsto \bar{f}$ and let $X$ be its real Banach subspace. 
Let $A: \mathcal{D}(A) \rightarrow \widetilde{X}$ be a linear operator of $\widetilde{X}$ with domain $\mathcal{D}(A) \subset \widetilde{X}$. Assume that $A$ satisfies the conditions:

$$
\begin{aligned}
u & \in \mathcal{D}(A) \text { is equivalent to } \operatorname{Re} u, \operatorname{Im} u \in \mathcal{D}(A), \\
\operatorname{Re} A u & =A(\operatorname{Re} u) \text { and } \operatorname{Im} A u=A(\operatorname{Im} u) \text { for } u \in \mathcal{D}(A) .
\end{aligned}
$$

These conditions can be described in terms of conjugate.

Proposition 1. In order that a linear operator $A: \mathcal{D}(A) \rightarrow \widetilde{X}$ satisfies (10) and (11), it is necessary and sufficient that A satisfies:

$$
\begin{gathered}
u \in \mathcal{D}(A) \quad \text { if and only if } \quad \bar{u} \in \mathcal{D}(A), \\
\overline{A u}=A \bar{u} \quad \text { for } u \in \mathcal{D}(A) .
\end{gathered}
$$

Proof. Let $A$ satisfy (10) and (11). Then, $u \in \mathcal{D}(A)$ if and only if $\operatorname{Re} u, \operatorname{Im} u \in \mathcal{D}(A)$; and these are obviously equivalent to $\bar{u} \in \mathcal{D}(A)$. Moreover, by (6), it holds for $u \in \mathcal{D}(A)$ that

$$
\begin{gathered}
\overline{A u}=\overline{\operatorname{Re} A u+i \operatorname{Im} A u}=\operatorname{Re} A u-i \operatorname{Im} A u, \\
A \bar{u}=A(\operatorname{Re} u-i \operatorname{Im} u)=A(\operatorname{Re} u)-i A(\operatorname{Im} u) .
\end{gathered}
$$

Hence, (11) implies (13).

Conversely, let $A$ satisfy (12) and (13). Then, $u \in \mathcal{D}(A)$ implies $u, \bar{u} \in \mathcal{D}(A)$; then, (5) shows that $\operatorname{Re} u, \operatorname{Im} u \in \mathcal{D}(A)$; hence, (10) is verified. Moreover, under (13),

$$
\begin{gathered}
\operatorname{Re} A u=(A u+\overline{A u}) / 2=(A u+A \bar{u}) / 2=A(\operatorname{Re} u), \\
\operatorname{Im} A u=(A u-\overline{A u}) / 2 i=(A u-A \bar{u}) / 2 i=A(\operatorname{Im} u) .
\end{gathered}
$$

Hence, (11) is verified.

We thus observed that (10) and (11) imply that $\mathcal{D}(A)=[\mathcal{D}(A) \cap X]+i[\mathcal{D}(A) \cap \underset{X}{\square}]$ and that $A$ maps $\mathcal{D}(A) \cap X$ into $X$. In this sense, we call $A$ a real linear operator of $\widetilde{X}$. In addition, we are naturally led to consider a part of $A$ restricted in the real subspace $X$ which is defined by

$$
\left\{\begin{array}{l}
\mathcal{D}\left(A_{\mid X}\right)=\mathcal{D}(A) \cap X, \\
A_{\mid X} u=A u
\end{array}\right.
$$

By (11), we then have

$$
A u=A_{\mid X}(\operatorname{Re} u)+i A_{\mid X}(\operatorname{Im} u) \quad \text { for } u \in \mathcal{D}(A) .
$$

Theorem 3. Let $A: \mathcal{D}(A) \rightarrow \widetilde{X}$ be a densely defined, closed linear operator of $\tilde{X}$ satisfying (10) and (11). Then, its part $A_{\mid X}$ in $X$ is a densely defined, closed real linear operator of $X$.

Proof. First, let us prove density of $\mathcal{D}\left(A_{\mid X}\right)$ in $X$. For any $f \in X$, there exists a sequence $u_{n} \in \mathcal{D}(A)$ such that $u_{n}$ converges to $f$ in $\widetilde{X}$. Then, $\operatorname{Re} u_{n} \in \mathcal{D}\left(A_{\mid X}\right)$ and $\operatorname{Re} u_{n} \rightarrow \operatorname{Re} f=f$ in $\widetilde{X}$ and of course in $X$. Hence, $\mathcal{D}\left(A_{\mid X}\right)$ is dense in $X$.

Second, let us prove closedness of $A_{\mid X}$. Consider sequences $u_{n} \in \mathcal{D}\left(A_{\mid X}\right)$ and $f_{n}=A u_{n}$ such that, as $n \rightarrow \infty, u_{n} \rightarrow u$ and $f_{n} \rightarrow f$ in $X$. By the closedness of $A, u \in \mathcal{D}(A)$ and $f=A u$. As $X$ is closed in $\widetilde{X}$ (due to Theorem 1), $u$ must be in $X$; thereby, $u \in \mathcal{D}\left(A_{\mid X}\right)$. Consequently, $f=A u=A_{\mid X} u$. 
We here remember the definition of sectorial operators of $\widetilde{X}$ (see [4]). A densely defined, closed linear operator $A$ is said to be a sectorial operator of $\widetilde{X}$ if its spectrum $\sigma(A)$ is contained in a sectorial domain

$$
\sigma(A) \subset \Sigma=\{\lambda \in \mathbb{C} ;|\arg \lambda|<\omega\}, \quad 0<\omega \leq \pi,
$$

and its resolvent satisfies the estimate

$$
\left\|(\lambda-A)^{-1}\right\| \leq \frac{M}{|\lambda|} \quad \text { for } \lambda \notin \Sigma,
$$

with some constant $M \geq 1$.

When a sectorial operator $A$ of $\widetilde{X}$ is a real linear operator, $A$ is called a real sectorial operator of $\widetilde{X}$. We can show various properties of real sectorial operators by the following theorems.

Theorem 4. Let $A$ be a real sectorial operator of $\widetilde{X}$. Then,

$$
\begin{gathered}
\lambda \in \rho(A) \quad \text { if and only if } \bar{\lambda} \in \rho(A) ; \\
\overline{(\lambda-A)^{-1} f}=(\bar{\lambda}-A)^{-1} \bar{f} \quad \text { for } \lambda \in \rho(A), f \in \widetilde{X} .
\end{gathered}
$$

In particular, when $\lambda \in \rho(A)$ is real, $(\lambda-A)^{-1}$ is a real operator and $\lambda$ belongs to the resolvent set $\rho\left(A_{\mid X}\right)$ of the part $A_{\mid X}$.

Proof. From (13), the relation $(\lambda-A) u=f$ for $u \in \mathcal{D}(A)$ and $f \in \tilde{X}$ is equivalent to $(\bar{\lambda}-A) \bar{u}=\bar{f}$. This then shows that $(17)$ holds true.

As seen, we have $(\lambda-A) u=f$ and $(\bar{\lambda}-A) \bar{u}=\bar{f}$ for $\lambda, \bar{\lambda} \in \rho(A)$. Thereby, $u=$ $(\lambda-A)^{-1} f$ and $\bar{u}=(\bar{\lambda}-A)^{-1} \bar{f}$. Hence, (18) is also shown.

When $\lambda \in \rho(A)$ is real, (18) means that $(\lambda-A)^{-1}$ satisfies (13). Hence, $(\lambda-A)^{-1}$ is a real operator.

Theorem 5. Let $A$ be a real sectorial operator of $\widetilde{X}$. Let, for $0<\theta<\infty, A^{\theta}$ be $i$ its fractional powers. Then, for every exponent $\theta, A^{\theta}$ is also a real operator.

Proof. The spectrum condition (15) implicitly means that $0 \notin \sigma(A)$. So, there exists $\delta>0$ such that $\{\lambda \in \mathbb{C} ;|\lambda| \leq \delta\} \subset \rho(A)$. We then introduce an integral contour $\Gamma=\Gamma_{-} \cup \Gamma_{0} \cup \Gamma_{+}$ such that $\Gamma_{ \pm}: \lambda=r e^{ \pm \omega i}, \delta \leq r<\infty$ and $\Gamma_{0}=\delta e^{\theta i},-\omega \leq \theta \leq \omega$. Its orientation is from $\infty e^{\omega i}$ to $\delta e^{\omega i}$, from $\delta e^{\omega i}$ to $\delta e^{-\omega i}$, and from $\delta e^{-\omega i}$ to $\infty e^{-\omega i}$. By definition, $A^{-\theta}$ is given by the integral

$$
A^{-\theta} f=\frac{1}{2 \pi i} \int_{\Gamma} \lambda^{-\theta}(\lambda-A)^{-1} f d \lambda \quad \text { for } f \in \tilde{X} .
$$

Taking the conjugate of each hand side, we obtain by (18) that

$$
\overline{A^{-\theta} f}=-\frac{1}{2 \pi i} \int_{\Gamma} \overline{\lambda^{-\theta}}(\bar{\lambda}-A)^{-1} \bar{f} d \bar{\lambda} .
$$

Here, $\overline{\lambda^{-\theta}}=e^{-\theta(\log |\lambda|-i \arg \lambda)}=(\bar{\lambda})^{-\theta}$. And, as $\lambda$ varies on $\Gamma$ in the positive sense, $\bar{\lambda}$ varies on the same contour $\Gamma$ in the negative sense. It therefore follows that

$$
\overline{A^{-\theta} f}=\frac{1}{2 \pi i} \int_{\Gamma} \lambda^{-\theta}(\lambda-A)^{-1} \bar{f} d \lambda=A^{-\theta} \bar{f} .
$$


This means that $A^{-\theta}$ satisfies (13). Thanks to Proposition $1, A^{-\theta}$ is a real operator (note that, as $A^{-\theta}$ is a bounded operator, (12) is automatically satisfied).

It is now easy to see that $A^{\theta}$ is real. Indeed, if $u \in \mathcal{D}\left(A^{\theta}\right)$, then there exists $f \in \widetilde{X}$ for which $u=A^{-\theta} f$ holds; therefore, $\bar{u}=A^{-\theta} \bar{f}$ and $\bar{u} \in \mathcal{D}\left(A^{\theta}\right)$; furthermore, $\overline{A^{\theta} u}=A^{\theta} \bar{u}$. It is clear that $\bar{u} \in \mathcal{D}\left(A^{\theta}\right)$ conversely implies $u=\overline{\bar{u}} \in \mathcal{D}\left(A^{\theta}\right)$. Hence, Proposition 1 is again available to $A^{\theta}$.

Consider a real sectorial operator $A$ of $\widetilde{X}$. As observed by Theorem 3 , its part $A_{\mid X}$ in $X$ is a densely defined, closed operator acting in $X$. Then, we can give a definition of fractional powers for $A_{\mid X}$. In fact, noting that $A^{\theta}$ is an operator from $\mathcal{D}\left(A^{\theta}\right) \cap X$ into $X$, we set

$$
\left[A_{\mid X}\right]^{\theta}=\left[A^{\theta}\right]_{\mid X} \quad \text { for any } 0<\theta<\infty
$$

with the domain

$$
\mathcal{D}\left(\left[A_{\mid X}\right]^{\theta}\right)=\mathcal{D}\left(A^{\theta}\right) \cap X
$$

Then,

$$
A^{\theta} u=\left[A_{\mid X}\right]^{\theta}(\operatorname{Re} u)+i\left[A_{\mid X}\right]^{\theta}(\operatorname{Im} u) \quad \text { for } u \in \mathcal{D}\left(A^{\theta}\right) .
$$

Theorem 6. Let $A$ be a real sectorial operator of $\widetilde{X}$ with angle $\omega<\frac{\pi}{2}$. Then, for the analytic semigroup $e^{-t A}(0 \leq t<\infty)$ generated by $-A, e^{-t A}$ is a real operator for any $0<t<\infty$.

Proof. Let $\Gamma$ be a similar integral contour used in the proof of Theorem 5. As well known, for $0<t<\infty$, the semigroup $e^{-t A}$ is given by

$$
e^{-t A} f=\frac{1}{2 \pi i} \int_{\Gamma} e^{-t \lambda}(\lambda-A)^{-1} f d \lambda, \quad f \in \widetilde{X}
$$

Taking the conjugate of each hand side, we obtain by the similar arguments as in the proof of Theorem 5 that

$$
\overline{e^{-t A} f}=\frac{1}{2 \pi i} \int_{\Gamma} \lambda^{-\theta}(\lambda-A)^{-1} \bar{f} d \lambda=e^{-t A} \bar{f} .
$$

This means that $e^{-t A}$ satisfies (13) of Proposition 1 and is a real operator of $\tilde{X}$. Consequently, $e^{-t A}$ is a real bounded operator acting on $X$.

Under the assumptions of Theorem 6 , we define a semigroup on $X$ generated by $-A_{\mid X}$ by the formula

$$
e^{-t A_{\mid X}}=\left[e^{-t A}\right]_{\mid X} \quad \text { for } 0<t<\infty .
$$

Then,

$$
e^{-t A} f=e^{-t A_{\mid X}}(\operatorname{Re} f)+i e^{-t A_{\mid X}}(\operatorname{Im} f) \quad \text { for } f \in \tilde{X} \text {. }
$$

Moreover,

$$
e^{-t A_{\mid X}} \cdot e^{-s A_{\mid X}}=e^{-(t+s) A_{\mid X}} \quad \text { on } X \text {. }
$$




\section{Interpolation and Real Subspaces}

Let $\widetilde{Z}$ and $\widetilde{X}$ be two complex Banach spaces such that $\widetilde{Z} \subset \widetilde{X}$ densely and continuously. We assume that $\widetilde{X}$ has a conjugation $f \mapsto \bar{f}$ on it. In addition, we assume that this conjugation is consistent to that of $\widetilde{Z}$ in the sense that

$$
\begin{gathered}
u \in \widetilde{Z} \quad \text { if and only if } \quad \bar{u} \in \widetilde{Z} \\
\|\bar{u}\|_{\widetilde{Z}}=\|u\|_{\widetilde{Z}} \quad \text { for } u \in \widetilde{Z} .
\end{gathered}
$$

For $0 \leq \theta \leq 1$, let $[\widetilde{X}, \widetilde{Z}]_{\theta}$ denote the complex interpolation space ( [11]). This space can also be decomposed into a sum of real part and imaginary part as in Theorem 2 .

Theorem 7. For any $0 \leq \theta \leq 1$,

$$
\begin{aligned}
& u \in[\widetilde{X}, \widetilde{Z}]_{\theta} \quad \text { if and only if } \bar{u} \in[\widetilde{X}, \widetilde{Z}]_{\theta}, \\
& \|\bar{u}\|_{[\widetilde{X}, \widetilde{Z}]_{\theta}}=\|u\|_{[\widetilde{X}, \widetilde{Z}]_{\theta}} \quad \text { for } u \in[\widetilde{X}, \widetilde{Z}]_{\theta} .
\end{aligned}
$$

Proof. Let $u \in[\widetilde{X}, \widetilde{Z}]_{\theta}$. By definition, there exists a holomorphic function $\Phi(z)$ defined in the band domain $G=\{z \in \mathbb{C} ; 0<\operatorname{Re} z<1\}$ with values in $\tilde{X}$, which is continuous and bounded on the closed domain $\bar{G}$, which takes its values on the straight line $\ell=$ $\{z=1+i y ;-\infty<y<\infty\}$ in $\widetilde{Z}$ with $\sup _{-\infty<y<\infty}\|\Phi(z)\|_{Z}<\infty$, and which takes a value $\Phi(\theta)=u$ at the point $z=\theta$. Then, the function $\Psi(z)=\overline{\Phi(\bar{z})}$ also possesses similar properties but $\Psi(\theta)=\bar{u}$. This then means that $\bar{u}$ also belongs to $[\widetilde{X}, \widetilde{Z}]_{\theta}$. Conversely, if $\bar{u} \in[\widetilde{X}, \widetilde{Z}]_{\theta}$, then $u=\overline{\bar{u}} \in[\widetilde{X}, \widetilde{Z}]_{\theta}$. Hence, (20) holds true.

We remember that

$$
\|u\|_{[\widetilde{X}, \widetilde{Z}]_{\theta}}=\inf \left\{\sup _{i y \in i \mathbb{R}}\|\Phi(i y)\|_{\widetilde{X}}+\sup _{1+i y \in \ell}\|\Phi(1+i y)\|_{\widetilde{Z}} ; \Phi(z)\right. \text { is }
$$

any holomorphic function in $G$ satisfying the properties mentioned above\}.

Then, (21) also follows immediately from this definition.

This theorem means that, when $\widetilde{X}$ has a conjugation $\bar{f} \mapsto f$ which is consistent with a conjugation on $\widetilde{Z}$ (i.e., (20) and (21)), the conjugation induces a conjugation on any interpolation space $[\widetilde{X}, \widetilde{Z}]_{\theta}$, too. We can then apply Theorems 1 and 2 to $[\widetilde{X}, \widetilde{Z}]_{\theta}$. Let $\widetilde{X}=X+i X$ and $\widetilde{Z}=Z+i Z$ be the decompositions for $\widetilde{X}$ and $\widetilde{Z}$, respectively, into real part and imaginary part. We naturally define

$$
[X, Z]_{\theta}=[\widetilde{X}, \widetilde{Z}]_{\theta} \cap X \quad \text { for any } 0 \leq \theta \leq 1
$$

Then, it holds true that

$$
[\widetilde{X}, \widetilde{Z}]_{\theta}=[X, Z]_{\theta}+i[X, Z]_{\theta} \quad \text { for any } 0 \leq \theta \leq 1
$$




\section{Triplet and Real Subspaces}

In this section, we want to consider a triplet of complex spaces $\widetilde{Z} \subset \widetilde{X} \subset \widetilde{Z}^{*}([12])$. Here, $\widetilde{X}$ is a complex Hilbert space, its inner product and norm being denoted by $(\cdot, \cdot)$ and $|\cdot|$, respectively. The space $\widetilde{Z}$ is a complex reflexive Banach space, its norm being denoted by $\|\cdot\|$, such that $\widetilde{Z}$ is densely and continuously embedded in $\widetilde{X}$. The third space $\widetilde{Z}^{*}$ is a complex adjoint space of $\widetilde{Z}$ with norm $\|\cdot\|_{*}$. There is a scaler product $\langle\cdot, \cdot\rangle$ between $\widetilde{Z}$ and $\widetilde{Z}^{*}$ which is sesquilinear and satisfies

$$
\begin{aligned}
& \|u\|=\sup _{\|\varphi\|_{*} \leq 1}|\langle u, \varphi\rangle| \quad \text { for } u \in \widetilde{Z}, \\
& \|\varphi\|_{*}=\sup _{\|u\| \leq 1}|\langle u, \varphi\rangle| \quad \text { for } \varphi \in \widetilde{Z}^{*}, \\
& \langle u, f\rangle=(u, f) \quad \text { for } u \in \widetilde{Z}, f \in \widetilde{X} .
\end{aligned}
$$

We assume that a conjugation $f \mapsto \bar{f}$ is defined on $\widetilde{X}$. Corollary 1 and Theorem 2 yield that $\widetilde{X}=X+i X$ with a real Hilbert space $X$. We assume in addition that the conjugation is consistent with that on $\widetilde{Z}$, i.e., $(20)$ and (21) being satisfied. Let $Z$ be the real subspace of $\widetilde{Z}$ induced by this conjugation. Then, the conjugation can be extended on the space $\widetilde{Z}^{*}$, too. In fact, due to $(9)$ we have

$$
\begin{aligned}
\|f\|_{*} & =\sup _{\|u\| \leq 1}|\langle u, f\rangle|=\sup _{\|u\| \leq 1}|(u, f)|=\sup _{\|u\| \leq 1}|\overline{(u, f)}| \\
& =\sup _{\|u\| \leq 1}|(\bar{u}, \bar{f})|=\sup _{\|\bar{u}\| \leq 1} \mid\langle\bar{u}, \bar{f}\rangle=\|\bar{f}\|_{*} \quad \text { for } f \in \widetilde{X}
\end{aligned}
$$

which shows that $f \mapsto \bar{f}$ is continuous in the norm $\|\cdot\|_{*}$, too. Density of $\widetilde{X}$ in $\widetilde{Z}^{*}$ then provides that the conjugation is extended on $\widetilde{Z}^{*}$ continuously. Of course, it holds true that

$$
\|\bar{\varphi}\|_{*}=\|\varphi\|_{*} \quad \text { for all } \varphi \in \widetilde{Z}^{*} .
$$

Moreover, from (9) and (27) it is verified that

$$
\overline{\langle u, \varphi\rangle}=\langle\bar{u}, \bar{\varphi}\rangle \quad \text { for } u \in \widetilde{Z}, \varphi \in \widetilde{Z}^{*} .
$$

Let $Z^{*}$ be the real subspace of $\widetilde{Z}^{*}$ induced by the conjugation on $\widetilde{Z}^{*}$. Then, (28) shows that the scaler product is real valued on $Z \times Z^{*}$.

Proposition 2. The two embeddings $Z \subset X \subset Z^{*}$ are dense and continuous. Moreover, (25) and (26) induce

$$
\begin{aligned}
& \|u\|=\sup _{\|\varphi\|_{*} \leq 1, \varphi \in Z^{*}}|\langle u, \varphi\rangle|, \quad \text { for } u \in Z, \\
& \|\varphi\|_{*}=\sup _{\|u\| \leq 1, u \in Z}|\langle u, \varphi\rangle|, \quad \text { for } \varphi \in Z^{*} .
\end{aligned}
$$

Proof. For $f \in X$, there exists a sequence $u_{n} \in \widetilde{Z}$ such that $u_{n} \rightarrow f$ in $\widetilde{X}$. Since $f \mapsto \operatorname{Re} f$ is continuous, it follows that $\operatorname{Re} u_{n} \in Z$ and $\operatorname{Re} u_{n} \rightarrow f$ in $X$; hence, $Z$ is dense in $X$. Similarly, we verify that $X$ is dense in $Z^{*}$. 
For $u \in Z$, there exists an element $\varphi \in \widetilde{Z}^{*}$ such that $\|\varphi\|_{*}=1$ and $\|u\|=\langle u, \varphi\rangle$. In view of (28), $\|u\|=\overline{\langle u, \varphi\rangle}=\langle u, \bar{\varphi}\rangle$. Therefore, we see that $\|u\|=\langle u, \operatorname{Re} \varphi\rangle$ together with $\|\operatorname{Re} \varphi\|_{*} \leq\|\varphi\|_{*} \leq 1$. Hence, $(29)$ is proved.

For $\varphi \in Z^{*}$, there exists a sequence $u_{n} \in \widetilde{Z}$ such that $\left\|u_{n}\right\| \leq 1$ and $\left\langle u_{n}, \varphi\right\rangle \rightarrow\|\varphi\|_{*}$. It is easy to see that it is the same for the sequence $\overline{u_{n}}$. Then, $\left\langle\operatorname{Re} u_{n}, \varphi\right\rangle \rightarrow\|\varphi\|_{*}$ together with $\left\|\operatorname{Re} u_{n}\right\| \leq\left\|u_{n}\right\| \leq 1$. Hence, (30) is proved.

This proposition has thus proved the following result for $Z \subset X \subset Z^{*}$.

Theorem 8. Let $Z, X$ and $Z^{*}$ be real subspaces introduced above. Then, $Z \subset X \subset Z^{*}$ make a triplet.

We finally remark an important property

$$
\left[Z^{*}, Z\right]_{\frac{1}{2}}=X
$$

In fact, it is known $([4,12])$ that

$$
\left[\widetilde{Z}^{*}, \widetilde{Z}\right]_{\frac{1}{2}}=\widetilde{X}
$$

Then, by (24), we have

$$
\left[Z^{*}, Z\right]_{\frac{1}{2}}=\left[\widetilde{Z}^{*}, \widetilde{Z}\right]_{\frac{1}{2}} \cap Z^{*}=\widetilde{X} \cap Z^{*}=X .
$$

\section{Real Sectorial Operators Determined from Sesquilinear Forms}

\subsection{Real Sesquilinear Forms}

Let $\widetilde{Z}$ and $\widetilde{X}$ be two complex Hilbert spaces with inner products $((\cdot, \cdot))$ and $(\cdot, \cdot)$ and norms $\|\cdot\|$ and $|\cdot|$, respectively, such that $\widetilde{Z} \subset \widetilde{X}$ densely and continuously. Then, there is a unique third Banach space $\widetilde{Z}^{*}$, its norm being denoted by $\|\cdot\|_{*}$, which composes a triplet

$$
\widetilde{Z} \subset \widetilde{X} \subset \widetilde{Z}^{*}
$$

The scaler product between $\widetilde{Z}$ and $\widetilde{Z}^{*}$ is denoted by $\langle\cdot, \cdot\rangle_{\widetilde{Z} \times \widetilde{Z}^{*}}$.

We assume that $\widetilde{X}$ has a conjugation $f \mapsto \bar{f}$ on it which is consistent with a conjugation on $\widetilde{Z}$. As seen in Section 4 , the conjugation induces a conjugation on $\widetilde{Z}^{*}$. Let $\widetilde{Z}=Z+$ $i Z, \tilde{X}=X+i X$ and $\widetilde{Z}^{*}=Z^{*}+i Z^{*}$ be the decompositions of $\widetilde{Z}, \widetilde{X}$ and $\tilde{Z}^{*}$, respectively. We know by Theorem 8 that these real subspaces also make a triplet

$$
Z \subset X \subset Z^{*}
$$

Consider a sesquilinear form $a(u, v)$ defined on $\widetilde{Z}$. We assume that $a(u, v)$ is continuous and coercive on $\widetilde{Z}$, i.e.,

$$
\begin{gathered}
|a(u, v)| \leq M\|u\|\|v\| \quad \text { for } u, v \in \widetilde{Z} \\
\operatorname{Re} a(u, u) \geq \delta\|u\|^{2} \quad \text { for } u \in \widetilde{Z}
\end{gathered}
$$

with some constants $M>0$ and $\delta>0$. 


\section{A. Yagi}

We furthermore assume that

$$
a(u, v) \text { is real valued for } u, v \in Z \text {. }
$$

Such a sesquilinear form is called a real sesquilinear form. It is possible to characterize the definition in terms of conjugate.

Proposition 3. $a(u, v)$ satisfies (34) if and only if

$$
\overline{a(u, v)}=a(\bar{u}, \bar{v}) \quad \text { for all } u, v \in \widetilde{Z} .
$$

Proof. Let (34) be satisfied. By sesquiliniarity, we have

$$
a(u, v)=a(\operatorname{Re} u, \operatorname{Re} v)+i a(\operatorname{Im} u, \operatorname{Re} v)-i a(\operatorname{Re} u, \operatorname{Im} v)+a(\operatorname{Im} u, \operatorname{Im} v) .
$$

Therefore,

$$
\begin{aligned}
\overline{a(u, v)} & =a(\operatorname{Re} u, \operatorname{Re} v)-i a(\operatorname{Im} u, \operatorname{Re} v)+i a(\operatorname{Re} u, \operatorname{Im} v)+a(\operatorname{Im} u, \operatorname{Im} v) \\
& =a(\operatorname{Re} u-i \operatorname{Im} u, \operatorname{Re} v-i \operatorname{Im} v)=a(\bar{u}, \bar{v}) .
\end{aligned}
$$

Conversely, let (35) be satisfied. If $u, v \in Z$, then $u=\bar{v}, v=\bar{v}$; therefore, $\overline{a(u, v)}=$ $a(u, v)$; hence, $a(u, v)$ is real.

According to the theory of variational methods ( $[12])$, the sesquilinear form $a(\cdot, \cdot)$ satisfying (32) and (33) defines a linear operator from $\widetilde{Z}$ into $\widetilde{Z}^{*}$ by the formula

$$
a(u, v)=\langle\mathcal{A} u, v\rangle_{\widetilde{Z}^{*} \times \widetilde{Z}} \quad \text { for } u, v \in \widetilde{Z} .
$$

It is also known as a linear operator of $\widetilde{Z}^{*}$ that $\mathcal{A}$ is a sectorial operator of angle $<\frac{\pi}{2}$ with the domain $\mathcal{D}(\mathcal{A})=\widetilde{Z}$. In addition, its part in $\widetilde{X}$, denoted by $A$, is defined by

$$
\left\{\begin{array}{l}
\mathcal{D}(A)=\{u \in \widetilde{Z} ; \mathcal{A} u \in \widetilde{X}\}, \\
A u=\mathcal{A} u
\end{array}\right.
$$

and is a sectorial operator of $\tilde{X}$ of angle $<\frac{\pi}{2}$.

The condition (34) in fact implies the following fact.

Theorem 9. Under (32), (33) and (34), let $\mathcal{A}$ and $A$ be sectorial operators of $\widetilde{Z}^{*}$ and $\widetilde{X}$, respectively, introduced above. Then, both $\mathcal{A}$ and $A$ are a real operator.

Proof. It follows from (36) that

$$
\overline{a(u, v)}=\overline{\langle\mathcal{A} u, v\rangle_{\widetilde{Z}^{*} \times \widetilde{Z}}}=\langle v, \mathcal{A} u\rangle_{\widetilde{Z} \times \widetilde{Z}^{*}} \quad \text { for } u, v \in \widetilde{Z} .
$$

Then, it is obtained by (28) and (35) that

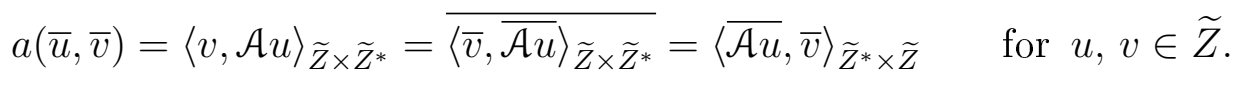


Meanwhile, by definition,

$$
a(\bar{u}, \bar{v})=\langle\mathcal{A} \bar{u}, \bar{v}\rangle_{\widetilde{Z}^{*} \times \widetilde{Z}} \quad \text { for } u, v \in \widetilde{Z} .
$$

Since $v \mapsto \bar{v}$ is onto $\widetilde{Z}$, we must have $\overline{\mathcal{A} u}=\mathcal{A} \bar{u}$ for any $u \in \widetilde{Z}$. Hence, $\mathcal{A}$ fulfills (13).

Similarly, since

$$
a(u, v)=(A u, v) \quad \text { for } u \in \mathcal{D}(A), v \in \widetilde{Z}
$$

we have

$$
\overline{a(u, v)}=\overline{(A u, v)} \quad \text { for } u \in \mathcal{D}(A), v \in \widetilde{Z} .
$$

Then, in view of (9), we can repeat the same argument to conclude that $A$ also fulfills (13).

We therefore arrive at the following result.

Corollary 2. Under (32), (33) and (34), $\mathcal{A}$ and $A$ are a real sectorial operator of $\widetilde{Z}^{*}$ and $\widetilde{X}$, respectively.

As shown, $\mathcal{A}$ is considered as a real linear operator from $Z$ onto $Z^{*}$, and $A$ as a real linear operator from $\mathcal{D}(A) \cap X$ onto $X$. In addition, these operators are nothing more than the operators $\mathcal{A}_{\mid Z^{*}}$ and $A_{\mid X}$, respectively.

It is known that $A$ satisfies $\left\|(\lambda-\mathcal{A})^{-1}\right\|_{\mathcal{L}(\widetilde{X})} \leq 1 /|\lambda|$ for $\lambda<0$ and this implies that $A$ is maximal accretive, i.e., $\operatorname{Re}(A u, u) \geq 0$ for $u \in \mathcal{D}(A)$. Then, $A$ possesses bounded purely imaginary powers $A^{i y}(-\infty<y<\infty)$, and consequently the domains of its fractional powers $A^{\theta}$ coincide with the interpolation spaces, that is,

$$
\mathcal{D}\left(A^{\theta}\right)=[\tilde{X}, \mathcal{D}(A)]_{\theta} \quad \text { for any } 0 \leq \theta \leq 1 .
$$

Thereby,

$$
\mathcal{D}\left(A^{\theta}\right) \cap X=[\tilde{X}, \mathcal{D}(A)]_{\theta} \cap X \quad \text { for any } 0 \leq \theta \leq 1 .
$$

In view of (19) and (24), it then follows that

$$
\mathcal{D}\left(\left[A_{\mid X}\right]^{\theta}\right)=[X, \mathcal{D}(A) \cap X]_{\theta}=\left[X, \mathcal{D}\left(A_{\mid X}\right)\right]_{\theta} \quad \text { for any } 0 \leq \theta \leq 1 .
$$

\subsection{Real Elliptic Operators}

We conclude this section with presenting an example of real sectorial operator which is determined from a real sesquilinear form.

Let $\Omega$ be a bounded domain in $\mathbb{R}^{n}$. Let $L_{2}(\Omega ; \mathbb{C})$ (resp. $H^{1}(\Omega ; \mathbb{C})$ ) be the complex $L_{2}$-space (resp. the complex Sobolev space of first order) in $\Omega$ with norm $\|\cdot\|_{L_{2}}$ (resp. $\left.\|\cdot\|_{H^{1}}\right)$. We consider a complex triplet

$$
H^{1}(\Omega ; \mathbb{C}) \subset L_{2}(\Omega ; \mathbb{C}) \subset H^{1}(\Omega ; \mathbb{C})^{*}
$$

where $H^{1}(\Omega ; \mathbb{C})^{*}$ is the adjoint space of $H^{1}(\Omega ; \mathbb{C})$. Let $f \mapsto \bar{f}$ be the complex conjugation on $L_{2}(\Omega)$ which obviously satisfies $(1) \sim(4)$ and is consistent with the conjugation on $H^{1}(\Omega ; \mathbb{C})$. Thereby, this induces a conjugation on $H^{1}(\Omega ; \mathbb{C})^{*}$, too. 
According to Theorem 2, the conjugation yields the decomposition of functions in $H^{1}(\Omega ; \mathbb{C}), L_{2}(\Omega ; \mathbb{C})$ and $H^{1}(\Omega ; \mathbb{C})^{*}$ into real part and imaginary part. But, it is nothing more than

$$
\left\{\begin{array}{c}
H^{1}(\Omega ; \mathbb{C})=H^{1}(\Omega ; \mathbb{R})+i H^{1}(\Omega ; \mathbb{R}) \\
L_{2}(\Omega ; \mathbb{C})=L_{2}(\Omega ; \mathbb{R})+i L_{2}(\Omega ; \mathbb{R}) \\
H^{1}(\Omega ; \mathbb{C})^{*}=H^{1}(\Omega ; \mathbb{R})^{*}+i H^{1}(\Omega ; \mathbb{R})^{*}
\end{array}\right.
$$

here $L_{2}(\Omega ; \mathbb{R})\left(\right.$ resp. $\left.H^{1}(\Omega: \mathbb{R})\right)$ is the real $L_{2}$-space (resp. the real Sobolev space of first order $)$ in $\Omega$ and $H^{1}(\Omega ; \mathbb{R})$ is the adjoint space of $H^{1}(\Omega ; \mathbb{R})$. As shown by Theorem 8 , we have a real triplet

$$
H^{1}(\Omega ; \mathbb{R}) \subset L_{2}(\Omega ; \mathbb{R}) \subset H^{1}(\Omega ; \mathbb{R})^{*}
$$

We then set $\widetilde{Z}=H^{1}(\Omega ; \mathbb{C})$ and $\widetilde{X}=L_{2}(\Omega ; \mathbb{C})$. Consequently, $Z=H^{1}(\Omega ; \mathbb{R})$ and $X=$ $L_{2}(\Omega ; \mathbb{R})$.

Consider a sesquilinear form

$$
a(u, v)=\sum_{j, k=1}^{n} \int_{\Omega} a_{j k}(x) D_{j} u(x) D_{k} \overline{v(x)} d x+\int_{\Omega} c(x) u(x) \overline{v(x)} d x
$$

defined on $\widetilde{Z}=H^{1}(\Omega ; \mathbb{C})$. We assume that

$$
\begin{gathered}
a_{j k} \in L_{\infty}(\Omega ; \mathbb{R}) \quad \text { for } 1 \leq j, k \leq n, \text { and } \quad c \in L_{\infty}(\Omega ; \mathbb{R}) ; \\
\sum_{j, k=1}^{n} a_{j k}(x) \xi_{j} \xi_{k} \geq \delta|\xi|^{2} \quad \text { for almost } \forall x \in \Omega \text { and } \forall \xi=\left(\xi_{1}, \ldots, \xi_{n}\right) \in \mathbb{R}^{n} ; \text { and } \\
c(x) \geq \delta \text { for almost } \forall x \in \Omega,
\end{gathered}
$$

here $\delta>0$ is some constant.

By (41), the form $a(u, v)$ satisfies (32). In the meantime, since

$$
\sum_{j, k=1}^{n} a_{j k}(x)\left(\xi_{j}+i \eta_{j}\right)\left(\overline{\xi_{k}+i \eta_{k}}\right)=\sum_{j, k=1}^{n} a_{j k}(x)\left[\xi_{j} \xi_{k}+\eta_{j} \eta_{k}+i\left(\xi_{k} \eta_{j}-\xi_{k} \eta_{j}\right)\right]
$$

(42) yields that

$$
\begin{aligned}
& \operatorname{Re}\left[\sum_{j, k=1}^{n} a_{j k}(x)\left(\xi_{j}+i \eta_{j}\right)\left(\overline{\xi_{k}+i \eta_{k}}\right)\right] \geq \delta\left(|\xi|^{2}+|\eta|^{2}\right) \\
& \qquad \text { for } \forall \xi+i \eta=\left(\xi_{1}+i \eta_{1}, \ldots, \xi_{n}+i \eta_{n}\right) \in \mathbb{C}^{n} .
\end{aligned}
$$

This together with (43) shows that $a(u, v)$ satisfies (33), too. So, by (36) and (37), we can define sectorial operators.

Let $\mathcal{A}$ be the associated linear operator in $H^{1}(\Omega ; \mathbb{C})^{*}$. Then, $\mathcal{A}$ is a sectorial operator of $H^{1}(\Omega ; \mathbb{C})^{*}$ with domain $\mathcal{D}(\mathcal{A})=H^{1}(\Omega ; \mathbb{C})$ and of angle $<\frac{\pi}{2}$. For $v \in \mathcal{C}_{0}^{\infty}(\Omega)\left(\subset H^{1}(\Omega ; \mathbb{C})\right)$, (40) is written as

$$
a(u, v)=\left\langle-\sum_{j, k=1}^{n} D_{k}\left[a_{j k}(x) D_{j} u\right]+c(x) u, v\right\rangle_{\mathcal{C}_{0}^{\infty}(\Omega) * \times \mathcal{C}_{0}^{\infty}(\Omega)} .
$$


Hence, (36) implies in the sense of distribution that

$$
\mathcal{A} u=-\sum_{j, k=1}^{n} D_{k}\left[a_{j k}(x) D_{j} u\right]+c(x) u \quad \text { in } \Omega .
$$

In $\Omega, \mathcal{A}$ is thus a realization of the elliptic differential operator $-\sum_{j, k=1}^{n} D_{k}\left[a_{j k}(x) D_{j}\right]+$ $c(x)$.

Next, let $A$ denote the part of $\mathcal{A}$ in $L_{2}(\Omega ; \mathbb{C})$. Then, $A$ is a sectorial operator of $L_{2}(\Omega ; \mathbb{C})$ of angle $<\frac{\pi}{2}$. If $u \in \mathcal{D}(A)$, then, since $(40)$ is written as

$$
a(u, v)=(\mathcal{A} u, v)+\sum_{j, k=1}^{n} \int_{\partial \Omega} a_{j k}(x) \nu(x)_{k} D_{j} u(x) D_{k} \overline{v(x)} d x
$$

where $\nu(x)=\left(\nu_{1}(x), \ldots, \nu_{n}(x)\right)$ is the outer normal vector of $\partial \Omega$ at $x \in \partial \Omega, u$ must implicitly satisfy the boundary conditions

$$
\sum_{j, k=1}^{n} a_{j k}(x) \nu_{k}(x) D_{j} u=0 \quad \text { on } \partial \Omega .
$$

In this sense, $A$ is a realization of $-\sum_{j, k=1}^{n} D_{k}\left[a_{j k}(x) D_{j}\right]+c(x)$ under the Neumann type boundary conditions $\sum_{j, k=1}^{n} a_{j k}(x) \nu_{k}(x) D_{j} u=0$ on $\partial \Omega$.

It is immediate to verify that (41) yields (34). Hence, Theorem 9 and Corollary 2 are available to the operators $\mathcal{A}$ and $A$ to conclude that $\mathcal{A}$ is a real sectorial operator of $H^{1}(\Omega ; \mathbb{C})^{*}$ and $A$ is a real sectorial operator of $L_{2}(\Omega ; \mathbb{C})$. Furthermore, in view of $(39)$, $\mathcal{A}_{\mid H^{1}(\Omega ; \mathbb{R})^{*}}$ is a densely defined, closed linear operator of $H^{1}(\Omega ; \mathbb{R})^{*}$ having the domain $H^{1}(\Omega ; \mathbb{R})$ and $A_{\mid L_{2}(\Omega ; \mathbb{R})}$ is a densely defined, closed linear operator of $L_{2}(\Omega ; \mathbb{R})$ having the domain $\mathcal{D}(A) \cap L_{2}(\Omega ; \mathbb{R})$.

In applications, it is often very important to know the domains of fractional powers $\mathcal{A}^{\theta}$ or $A^{\theta}$ for $0<\theta<1$. Especially, for $\theta=\frac{1}{2}$, we wonder if $\mathcal{D}\left(\mathcal{A}^{\frac{1}{2}}\right)=L_{2}(\Omega ; \mathbb{C})$ or if $\mathcal{D}\left(A^{\frac{1}{2}}\right)=H^{1}(\Omega ; \mathbb{C})$. Such a problem is called the square root problem, however, the answer is already known to be no in general (although (31) and (38) are the case). We have to restrict the class of sesquilinear forms to handle to that of, for example, symmetric forms. So, in addition to (41) and (42), let us assume that

$$
a_{j k}(x)=a_{k j}(x) \quad \text { for } 1 \leq j, k \leq n,
$$

which implies that $\overline{a(u, v)}=a(v, u)$ for $u, v \in H^{1}(\Omega ; \mathbb{C})$. Then, $\mathcal{A}^{*}=\mathcal{A}$ and hence $A^{*}=A$; in this way, $A$ is a positive definite self-adjoint operator of $L_{2}(\Omega ; \mathbb{C})$. Therefore, $a(u, u)=$ $(A u, u)=\left\|A^{\frac{1}{2}} u\right\|_{L_{2}}^{2}$ for $u \in \mathcal{D}(A)$. Furthermore, $\delta\|u\|_{H^{1}}^{2} \leq\left\|A^{\frac{1}{2}} u\right\|_{L_{2}}^{2} \leq M\|u\|_{H^{1}}^{2}$ for $u \in$ $\mathcal{D}(A)$. Finally, we conclude that $\mathcal{D}\left(A^{\frac{1}{2}}\right)=H^{1}(\Omega ; \mathbb{C})$. Due to $(38)$, it is obtained that

$$
\mathcal{D}\left(A^{\theta}\right)=\left[\mathcal{D}\left(A^{0}\right), \mathcal{D}\left(A^{\frac{1}{2}}\right)\right]_{2 \theta}=\left[L_{2}(\Omega ; \mathbb{C}), H^{1}(\Omega ; \mathbb{C})\right]_{2 \theta}=H^{2 \theta}(\Omega ; \mathbb{C}) \quad \text { for } 0 \leq \theta \leq \frac{1}{2} .
$$

Consequently, taking intersections with $L_{2}(\Omega ; \mathbb{R})$ for both hand sides, we verify by $(19)$ and (24) that

$$
\mathcal{D}\left(\left[A_{\mid L_{2}(\Omega ; \mathbb{R})}\right]^{\theta}\right)=H^{2 \theta}(\Omega ; \mathbb{R}) \quad \text { for } 0 \leq \theta \leq \frac{1}{2},
$$

where $H^{2 \theta}(\Omega ; \mathbb{R})$ is the real Sobolev spaces with the exponent $2 \theta$. 
Under $(44), A^{\frac{1}{2}}$ is an isomorphism from $H^{1}(\Omega ; \mathbb{C})$ onto $L_{2}(\Omega ; \mathbb{C})$. So, the purely imaginary powers of $\mathcal{A}$ are expressed by $\mathcal{A}^{i y}=\mathcal{A} A^{-\frac{1}{2}} A^{i y} A^{\frac{1}{2}} \mathcal{A}^{-1}(-\infty<y<\infty)$ and are bounded operators on $H^{1}(\Omega ; \mathbb{C})^{*}$ (since $A^{i y} \in \mathcal{L}\left(L_{2}(\Omega ; \mathbb{C})\right.$ ) for any $\left.-\infty<y<\infty\right)$. Hence, $\mathcal{D}\left(\mathcal{A}^{\frac{1}{2}}\right)=\left[\mathcal{D}\left(\mathcal{A}^{0}\right), \mathcal{D}(\mathcal{A})\right]_{\frac{1}{2}}=L_{2}(\Omega ; \mathbb{C})$ due to $(31)$. Furthermore, for $\frac{1}{2} \leq \theta \leq 1$,

$$
\mathcal{D}\left(\mathcal{A}^{\theta}\right)=\left[\mathcal{D}\left(\mathcal{A}^{\frac{1}{2}}\right), \mathcal{D}(\mathcal{A})\right]_{2 \theta-1}=\left[L_{2}(\Omega ; \mathbb{C}), H^{1}(\Omega ; \mathbb{C})\right]_{2 \theta-1}=H^{2 \theta-1}(\Omega ; \mathbb{C}) .
$$

Consequently, taking intersections with $H^{1}(\Omega ; \mathbb{R})^{*}$ for both hand sides, we verify by $(19)$ and (24) that

$$
\mathcal{D}\left(\left[\mathcal{A}_{\mid H^{1}(\Omega ; \mathbb{R})^{*}}\right]^{\theta}\right)=H^{2 \theta-1}(\Omega ; \mathbb{R}) \quad \text { for } \frac{1}{2} \leq \theta \leq 1 .
$$

It is equally possible to set $\widetilde{Z}=H_{0}^{1}(\Omega ; \mathbb{C})\left(\right.$ instead of $\left.H^{1}(\Omega ; \mathbb{C})\right)$, where $H_{0}^{1}(\Omega ; \mathbb{C})$ is a completion of the space $\mathcal{C}_{0}^{\infty}(\Omega ; \mathbb{C})$ by the $H^{1}$-Sobolev norm. Then, we have a triplet

$$
H_{0}^{1}(\Omega ; \mathbb{C}) \subset L_{2}(\Omega ; \mathbb{C}) \subset H^{-1}(\Omega ; \mathbb{C})\left(=H_{0}^{1}(\Omega ; \mathbb{C})^{*}\right)
$$

The sesquilinear form $(40)$ is considered on $H_{0}^{1}(\Omega ; \mathbb{C})$ under the same assumptions $(41)$, (42) and (43). Then, the operator $\mathcal{A}$ determined by (36) becomes a real sectorial operator of $H^{-1}(\Omega ; \mathbb{C})$ of angle $<\frac{\pi}{2}$, and its part $A$ determined by $(37)$ is a real sectorial operator of $L_{2}(\Omega ; \mathbb{C})$ of angle $<\frac{\pi}{2}$. In the meantime, $\mathcal{A}$ is a realization of the elliptic differential operator $-\sum_{j, k=1}^{n} D_{k}\left[a_{j k}(x) D_{j}\right]+c(x)$ under the Dirichlet boundary conditions $u=0$ on $\partial \Omega$. In addition, $\mathcal{A}_{\mid H^{-1}(\Omega ; \mathbb{R})}$ is a densely defined, closed real linear operator of $H^{-1}(\Omega ; \mathbb{R})$, and $A_{\mid L_{2}(\Omega ; \mathbb{R})}$ is a densely defined, closed real linear operator of $L_{2}(\Omega ; \mathbb{R})$.

Furthermore, assume that (44) is satisfied. Then, $\mathcal{A}^{*}=\mathcal{A}$ and $A$ is a positive definite self-adjoint operator of $L_{2}(\Omega ; \mathbb{C})$. The similar arguments as above yield analogous results to (45) and (46) which characterize the domains $\mathcal{D}\left(\left[\mathcal{A}_{\mid H^{-1}(\Omega ; \mathbb{R})}\right]^{\theta}\right)$ or $\mathcal{D}\left(\left[A_{\mid L_{2}(\Omega ; \mathbb{R})}\right]^{\theta}\right)$ of fractional powers of $\mathcal{A}_{\mid H^{-1}(\Omega ; \mathbb{R})}$ or $A_{\mid L_{2}(\Omega ; \mathbb{R})}$, respectively.

\section{Real Sectorial Operators Obtained by Complexfication}

This section is devoted to considering how to construct real sectorial operators from real linear operators.

Let $\widetilde{X}$ be a complex Banach space with norm $\|\cdot\|$ and with conjugation $f \mapsto \bar{f}$, and let $\widetilde{X}=X+i X$ be the decomposition into real and imaginary parts. Let a real linear operator $A: \mathcal{D}(A) \rightarrow X$ be given with domain $\mathcal{D}(A) \subset X$. By the formula

$$
A(u+i v)=A u+i A v \quad \text { for } u+i v \in X+i X=\tilde{X},
$$

we can extend $A$ to a complex linear operator in $\widetilde{X}$ with the domain $\mathcal{D}(A)+i \mathcal{D}(A)$. Indeed, we verify that

$$
\begin{aligned}
A\left(u_{1}+i v_{1}+u_{2}+i v_{2}\right)=A & \left(u_{1}+u_{2}\right)+i A\left(v_{1}+v_{2}\right) \\
& =A\left(u_{1}+i v_{1}\right)+A\left(u_{2}+i v_{2}\right), \quad u_{k}+i v_{k} \in \tilde{X}, k=1,2,
\end{aligned}
$$

and

$$
\begin{array}{r}
A((a+b i)(u+i v))=A(a u-b v+i(b u+a v))=A(a u-b v)+i A(b u+a v) \\
=(a+b i) A(u+i v), \quad a+b i \in \mathbb{C}, u+i v \in \tilde{X} .
\end{array}
$$


Theorem 10. If $A$ is a densely defined, closed linear operator of $X$, then it is the same for the extended operator $A$ in $\widetilde{X}$.

Proof. The proof is quite direct if we notice (8).

Consider a densely defined, closed linear operator $A: \mathcal{D}(A) \rightarrow X$ of $X$. We set

$$
\begin{aligned}
\rho_{\mathbb{R}}(A)=\left\{(\xi, \eta) \in \mathbb{R}^{2} ;\left[(\xi-A)^{2}+\eta^{2}\right]: \mathcal{D}\left(A^{2}\right) \rightarrow X\right. \text { is one-to-one and onto, } \\
\text { and } \left.\left[(\xi-A)^{2}+\eta^{2}\right]^{-1} \text { is a bounded operator on } X\right\} .
\end{aligned}
$$

We set also $\sigma_{\mathbb{R}}(A)=\mathbb{R}^{2}-\rho_{\mathbb{R}}(A)$. Assume that

$$
\sigma_{\mathbb{R}}(A) \subset \Sigma=\left\{(\xi, \eta) \in \mathbb{R}^{2} ;|\arg (\xi, \eta)|<\omega\right\}, \quad 0<\exists \omega \leq \pi,
$$

and that the inverse $\left[(\xi-A)^{2}+\eta^{2}\right]^{-1}$ satisfies the estimate

$$
\begin{aligned}
\left\|(\xi-A)\left[(\xi-A)^{2}+\eta^{2}\right]^{-1}\right\|+\left\|\eta\left[(\xi-A)^{2}+\eta^{2}\right]^{-1}\right\| & \\
& \leq \frac{M}{|\xi|+|\eta|} \quad \text { for }(\xi, \eta) \notin \Sigma,
\end{aligned}
$$

with some constant $M \geq 1$.

These conditions are then shown to be sufficient conditions in order that $A$ is a real sectorial operator.

Theorem 11. If a densely defined, closed real linear operator $A$ of $X$ satisfies (47) and (48), then $A$ is a real sectorial operator of $\tilde{X}$.

Proof. For given $\xi+i \eta \in \mathbb{C}$ and $f+i g \in X+i X$, consider the equation

$$
[(\xi+i \eta)-A](u+i v)=f+i g
$$

for $u+i v \in \mathcal{D}(A)+i \mathcal{D}(A)$. This is rewritten in the form

$$
\left(\begin{array}{cc}
\xi-A & -\eta \\
\eta & \xi-A
\end{array}\right)\left(\begin{array}{l}
u \\
v
\end{array}\right)=\left(\begin{array}{l}
f \\
g
\end{array}\right)
$$

Therefore, if $(\xi, \eta) \in \rho_{\mathbb{R}}(A)$, then this equation has a unique solution given by

$$
\left(\begin{array}{l}
u \\
v
\end{array}\right)=\left(\begin{array}{cc}
\xi-A & \eta \\
-\eta & \xi-A
\end{array}\right)\left[(\xi-A)^{2}+\eta^{2}\right]^{-1}\left(\begin{array}{l}
f \\
g
\end{array}\right),
$$

i.e., $\xi+i \eta \in \rho(A)$. Moreover, we verify that, if the estimate (48) holds true for $(\xi, \eta) \in$ $\rho_{\mathbb{R}}(A)$, then the estimate (16) holds for the corresponding $\xi+i \eta$. Hence, (48) implies (16).

Acknowledgements. The author is supported by Grant-in-Aid for Scientific Research (No. 26400166) of the Japan Society for the Promotion of Science. 


\title{
References
}

1. Krein S.G. Linear Differential Equations in Banach Space. American Mathematical Society, 1971.

2. Tanabe H. Equations of Evolution. London, Pitman, 1979.

3. Favini A., Yagi A. Degenerate Differential Equations in Banach Spaces. N.Y., Marcel-Dekker, 1999 .

4. Yagi A. Abstract Parabolic Evolution Equations and Their Applications. Berlin, Springer, 2010. DOI: $10.1007 / 978-3-642-04631-5$

5. Azizi S., Mola G., Yagi A. Longtime Convergence for Epitaxial Growth Model under Dirichlet Conditions, Osaka Journal of Mathematics. Accepted for publication.

6. Grasselli M., Mola G., Yagi A. On the Longtime Behavior of Solutions to a Model for Epitaxial Growth. Osaka Journal of Mathematics, 2011, vol. 48, no. 4, pp. 987-1004.

7. Chill R. The Lojasiewicz - Simon Gradient Inequality on Hilbert Spaces. Proceedings 5th European-Maghrebian Workshop on Semigroup Theory, Evolution Equations and Applications, 2006, pp. 25-36.

8. Haraux A., Jendoubi M.A. The Lojasiewicz Gradient Inequality in the Infinite-Dimensional Hilbert Space Framework. Journal of Functional Analysis, 2011, vol. 260, issue 9, pp. 2826-2842. DOI: 10.1016/j.jfa.2011.01.012

9. Brezis H. Functional Analysis, Sobolev Spaces and Partial Differential Equations. N.Y., Springer, 2011. DOI: 10.1007/978-0-387-70914-7

10. Yosida K.Functional Analysis. Berlin, Heidelberg, N.Y., Springer-Veglar, 1980.

11. Triebel H. Interpolation Theory, Function Spaces, Differential Operators. Amsterdam, N.Y., Oxford, North-Holland, 1978.

12. Dautray R., Lions J.L. Mathematical Analysis and Numerical Methods for Science and Technology, Vol. 2. Berlin, Springer-Verlag, 1988.

Received December 14, 2016

УДК 517.9

DOI: $10.14529 / \mathrm{mmp} 170106$

\section{ДЕЙСТВИТЕЛЬНЫЕ СЕКТОРИАЛЬНЫЕ ОПЕРАТОРЫ}

\section{A. Яги}

\begin{abstract}
Секториальные операторы, которые действуют в комплексных банаховых пространствах и отображают действительные подпространства в себя, называются действительными секториальными операторами. Эти операторы уже неявно используются при изучении различных диффузионных уравнений. Между тем, в теории Лоясевича Саймона, которая обеспечивает сходимость решений к стационарным решениям, действительнозначные функции Ляпунова играют важную роль. Для того чтобы создать общие методы изучения задач сходимости на основе теории Лоясевича - Саймона, целесообразно дать явное определение действительных секториальных операторов и показать их основные свойства, которые наследуются от комплексньх секториальньх операторов.
\end{abstract}

Ключевые слова: секториальные операторы; дробные степени оператора; дифференциальные операторь.

Атсуши Яги, кафедра прикладной физики, Университет Осаки (г. Суйта, Осака, Япония), yagi@ap.eng.osaka-u.ac.jp.

Поступила в редакиию 14 декабря 2016 2. 